\title{
An Apolipoprotein CIII-Derived Peptide, Hatktak, Activates Macromolecular Activators of Phagocytosis from Platelets (MAPPs)
}

\author{
Haruhiko Sakamoto, Masaki Ueno, Wu Bin, Yumiko Nagai, \\ Kouichi Matsumoto, Takao Yamanaka and Sumiko Tanaka
}

Additional information is available at the end of the chapter

http://dx.doi.org/10.5772/48173

\section{Introduction}

When thrombi are formed, infiltration of leukocytes including neutrophils and macrophages follows in and around the thrombi. On the other hand, in inflammatory lesions where infiltration of leukocytes is observed, thrombi are often observed. When thrombi are formed, activated platelets release a lot of substances while adhering to subendothelial connective tissues, recruiting other platelets and aggregating together. In these regions with thrombus formation or platelet activation, it is possible that platelets affect leukocytic function via the action of the released products, since the substances released from platelets include factors that activate [1-14] and suppress [15-22] neutrophilic functions.

In experiments using human platelets and neutrophils, we found that platelets release several neutrophilic phagocytosis activators. Among substances released from activated platelets, ATP and ADP have been reported to activate $\mathrm{iC} 3 \mathrm{~b}$ receptor-mediated phagocytosis $[1,2,23]$, and some prostaglandins including PGE2, PGF2 $\alpha$ and thromboxane B2 and macromolecular activators of phagocytosis from platelets (MAPPs) activate Fc $\gamma$ receptormediated phagocytosis.

MAPPs have two subsets, l-MAPP $\left(3 \times 10^{5} \mathrm{Da}\right)$ and s-MAPP $\left(1.5 \times 10^{5} \mathrm{Da}\right)[24,25]$. Platelets stored in the form of platelet-rich plasma lose the capacity to release MAPPs but recover it if incubated with the plasma-derived precursors of MAPPs (precursors of 1-MAPP and sMAPP, $3 \times 10^{5} \mathrm{Da}$ and $1.5 \times 10^{5} \mathrm{Da}$, respectively) and thrombin in the presence of $\mathrm{Ca}^{++}$[26]. It was suggested that the loss of platelet ability to release MAPPs is due to escape of the precursors and thrombin during storage with CPD (citrate-phosphate-dextrose) solution. It is possible to produce MAPPs using stored platelet-derived lysate by stimulation with 
thrombin or trypsin in the presence of the precursors [27]. It has also been suggested that the precursors of 1-MAPP and s-MAPP are polymerized transferrins, probably of tetrameric and dimeric forms, respectively [28].

As for the production of MAPPs in platelets, it was suggested that GP Ib $\alpha$-bound thrombin reacts with a high-molecular-weight substance (HMW activator) to release a low-molecularweight substance (LMW activator) [29], which can produce MAPPs from the precursors directly.

In an ultracentrifugation study of the platelet lysate, the HMW activator activity was observed in the HDL fraction. Anti-apolipoprotein A1 antibody abolished the HMW activator function from the HDL fraction of the platelet lysate. These findings suggest that the HMW activator belongs to HDL. In an affinity chromatography study of the protein obtained from the HDL-rich fraction of the platelet lysate using an anti-apolipoprotein CIII (Apo CIII) column, it was suggested that LMW activator is derived from Apo CIII. In fact, it was observed that the commercially available Apo CIII could produce LMW activator by the activity of thrombin [30].

The purpose of this study was to determine the structure of the LMW activator.

\section{Materials and methods}

This study was approved by the local institutional review board of our university hospital. The procedures followed were in accordance with the Helsinki Declaration of 1975, as revised in 1983.

\subsection{Phagocytosis experiment}

The phagocytosis experiments were performed according to a method previously described [30]. Briefly, neutrophils were separated from heparinized venous blood from healthy volunteers by centrifugation on MonoPoly resolving medium (ICN Biochemicals Japan, Tokyo, Japan). After washing of neutrophils in phosphate-buffered saline (PBS) (Sigma, St. Louis, MO), one thousand neutrophils in $5 \mu \mathrm{l}$ of PBS were attached to the surface of a Terasaki microplate well (Nunc, Roskilde, Denmark) by centrifugation at $160 \mathrm{~g}$ for 2 minutes. Then, stimulation was performed with $10 \mu \mathrm{l}$ of the test material in PBS supplemented with $1 \%$ bovine serum albumin (Sigma) (PBS-BSA) at $37{ }^{\circ} \mathrm{C}$ for 15 minutes. After washing, the neutrophils were incubated with 5,000 sheep red blood cells (SRBCs) in $5 \mu$ l of RPMI 1640 medium supplemented with 1\% BSA and a one-hundredth volume of anti-SRBC rabbit IgG at $37{ }^{\circ} \mathrm{C}$ for 25 minutes under $5 \% \mathrm{CO}_{2}$. After lysing the unphagocytosed SRBCs using a Trisbuffered ammonium chloride solution [31] and fixation with 1\% glutaraldehyde (Sigma) in PBS, the phagocytosed SRBCs were counted under a light microscope.

All phagocytosis experiments were performed in triplicate. Mean numbers of ingested SRBCs per neutrophil in a well were calculated. Then, means of three wells were obtained. The results are expressed as phagocytic indices normalized by the phagocytic activity of the PBS-BSA control taken as 100. 


\subsection{Production of MAPPs in vitro}

\subsubsection{Preparation of the precursors of MAPPs using holo-transferrin}

Precursors of MAPPs were produced artificially using holo-transferrin (Sigma) according to a method described previously [28, 30]. Briefly, the peak fraction of holo-transferrin in Superdex 200 (GE Healthcare UK Ltd., Buckinghamshire, England) gel filtration was incubated on ice for $15 \mathrm{~min}$ with $0.2 \%$ glutaraldehyde (Sigma) to induce polymerization [32], and subjected to filtration again. The fractions obtained at the same elution volumes as the conventional 1-MAPP (tetramer transferrin-rich fraction) and s-MAPP (dimer transferrinrich fraction) were adjusted to an optical density of 0.04 at $280 \mathrm{~nm}$ with PBS and used as precursors of 1-MAPP and s-MAPP, respectively.

\subsubsection{Preparation of $L M W$ activators}

Apo CIII (Chemicon International Inc., Temecula, CA) was adjusted to a concentration of 10 $\mu \mathrm{g} / \mathrm{ml}$ in PBS and incubated with $0.1 \mathrm{unit} / \mathrm{ml}$ human thrombin (thrombin) (Sigma) or 1 unit/ml bovine trypsin (trypsin) (Sigma) at $37^{\circ} \mathrm{C}$ for 30 minutes, and then applied on a PD10 column (GE Healthcare UK Ltd.) to obtain the low-molecular-weight fraction, which was serially diluted $\mathrm{x} 10$ and used as the LMW activator.

Another Apo CIII $(10 \mu \mathrm{g} / \mathrm{ml})$ was incubated with 0.1 unit $/ \mathrm{ml}$ thrombin or 1 unit $/ \mathrm{ml}$ trypsin and gel-filtered through a Superdex peptide column (GE Healthcare UK Ltd.) (elution buffer, PBS; elution speed, $0.5 \mathrm{ml} /$ minute; fraction volume, $1 \mathrm{ml}$ each) to obtain LMW activator fraction.

Samples of the low-molecular-weight fraction of variously prepared platelet-related materials including platelet release products and platelet lysate (see below for details) were obtained using PD-10 columns.

Some peptides associated with Apo CIII were synthesized artificially, and the activity as an LMW activator was examined.

\subsubsection{Preparation of MAPPs using LMW activators and precursors of MAPPS}

To form MAPPs, $1 \mathrm{ml}$ of appropriately diluted LMW activator derived from Apo CIII, platelet release products or artificially produced peptides was incubated with $10 \mu \mathrm{l}$ of precursor of 1-MAPP or s-MAPP at $37^{\circ} \mathrm{C}$ for 30 minutes.

\subsection{Preparation of platelet release products using platelets from platelet-rich plasma}

To prepare fresh and stored platelets, heparinized venous blood was mixed with $13 \mathrm{vol} \%$ citrate-phosphate-dextrose (CPD) solution and centrifuged at $60 \mathrm{~g}$ for 20 minutes at $20{ }^{\circ} \mathrm{C}$. The platelet-rich plasma, which was contaminated by less than one erythrocyte per 1,000 platelets, was transferred into another plastic tube and stored at $20{ }^{\circ} \mathrm{C}$ in a water bath with agitation once a second for 0,72 or 120 hours. 
These platelets were washed twice in PBS supplemented with $6.7 \mathrm{mM}$ EDTA and twice in PBS, and adjusted to a concentration of $4 \times 10^{5} / \mu$ l. The platelet suspension was stimulated with $0.1 \mathrm{unit} / \mathrm{ml}$ thrombin at $37^{\circ} \mathrm{C}$ for one minute in the presence or absence of $4 \mathrm{mEq} / 1 \mathrm{Ca}^{++}$, then cooled immediately on an ice-water bath and centrifuged at $960 \mathrm{~g}$ for 15 minutes at $4{ }^{\circ} \mathrm{C}$ to obtain the platelet release products in the supernatant.

\subsection{Preparation of platelet lysate}

Platelet suspensions obtained from platelet-rich plasma as described above were frozen at $15{ }^{\circ} \mathrm{C}$, thawed and centrifuged at $1,500 \mathrm{~g}$ for 30 minutes. The supernatant was used as the platelet lysate.

\subsection{Peptide synthesis}

Peptides such as S1-K21, H18-R40, H18-K24, H18-K21 and T22-K24 of Apo CIII were synthesized by Sawady Technology (Tokyo, Japan). Other peptides that contain part or all of H18-K24 (HATKTAK) of Apo CIII were produced by Thermo Fisher Scientific GmbH (Ulm, Germany).

\subsection{Inhibition of the LMW activator activity by anti-HATKTAK rabbit IgG antibody}

Antibody against HATKTAK was raised in a rabbit by intracutaneous injection of keyholelimpet-conjugated CHATKTAK (6 injections at 2-week intervals) by Sigma Aldrich Japan (Ishikari city, Japan). The IgG antibody was refined using a protein A column. The control $\mathrm{IgG}$ antibody was obtained from the same rabbit at day 0 of immunization.

By indirect enzyme-linked immunosorbent assay (ELISA) [33], specificity of the antiHATKTAK rabbit antibody was examined. $100 \mu$ l of antigens involving Apo CIII and Apo CIII-related peptides $(1 \mu \mathrm{g} / \mathrm{ml}$ in PBS) was immobilized to each well of a polystyrene microplate by incubation at $4{ }^{\circ} \mathrm{C}$ overnight. 1000-fold-diluted anti-HATKTAK rabbit antibody or 1000-fold-diluted anti-Apo CIII gout IgG (Gene Tex, Inc., San Antonio, TX) was used as the primary antibody. Peroxidase-conjugated anti-rabbit IgG (Fab') or anti-goat IgG $\left(\mathrm{Fab}^{\prime}\right)$ (Histofine simple stain MAX-PO(R) or $-\mathrm{PO}(\mathrm{G})$, respectively, Nichirei Bioscience, Tokyo, Japan) was used as the secondary antibody. Substrate solution consisted of $10 \mathrm{ml}$ of $0.5 \mathrm{M}$ citrate buffer, $10 \mathrm{ml}$ of $0.3 \%$ hydrogen peroxide and $10 \mathrm{mg}$ of orthophenylene diamine (Wako, Osaka, Japan). 2 M sulfuric acid was used as a stopping solution. Absorbance was determined at $450 \mathrm{~nm}$ in a model 550 microplate reader (Bio-Rad, Tokyo, Japan).

To examine the effect of anti-HATKTAK antibody on an LMW activator, a $\times 10^{2}$-diluted lowmolecular-weight fraction of the platelet release products prepared from fresh platelet- rich plasma and a $\times 10^{6}$-diluted one from platelet-rich plasma stored for 120 hours were prepared. Then, they were incubated with a one-hundredth volume of anti-HATKTAK IgG or control $\mathrm{IgG}$ at room temperature for $30 \mathrm{~min}$, applied to a protein A column to remove the immune complexes and the residual antibodies, and used as the source of the LMW activator. 


\subsection{Ion exchange chromatography}

Cation exchange chromatography was performed using a MONO S HR5/5 column (GE Healthcare UK Ltd.). Samples were dissolved in $20 \mathrm{mM}$ Tris- $\mathrm{HCl}, \mathrm{pH} 8.0$ (buffer A). The adsorbed materials were recovered by elution with a linear increase in buffer B (buffer $\mathrm{A}+1$ $\mathrm{M} \mathrm{NaCl})$.

\subsection{Assessment of peptide concentrations using the o-phthalaldehyde (OPA) fluorescent method}

Peptide concentrations were assessed according to the OPA fluorescent method with intact protein [34]. Fluorescence was measured with an excitation wavelength of $340 \mathrm{~nm}$ and an emission wavelength of $450 \mathrm{~nm}$ using a spectrofluorometer (FP-6300, JASCO, Tokyo, Japan).

\subsection{Mass spectrometry}

Matrix-associated laser desorption/ionization time-of-flight mass spectrometry was performed with a Voyager System 4314 (Applied Biosystems, Foster City, CA) in the reflector time-offlight configuration at an acceleration voltage of $25 \mathrm{kV}$ with delayed ion extraction. Samples were diluted 1:1 with a freshly prepared matrix solution consisting of $10 \mathrm{mg} / \mathrm{ml} \alpha$-cyano-4hydroxycinnamic acid (Sigma) in $20 \%$ acetonitrile with $0.1 \%$ trifluoroacetic acid. Aliquots of 1 $\mu \mathrm{l}$ were deposited on a metallic sample holder and analyzed immediately after drying in a stream of air. Mass scale calibration was performed externally.

\subsection{Immunohistochemistry}

Coagulation of the fresh peripheral blood taken without anticoagulants was induced in a glass tube for 3 hours. This coagulum was formalin-fixed, and paraffin sections of $3 \mu \mathrm{m}$ thickness were prepared. After deparaffinization and hydration, antigen retrieval was performed by heating in $10 \mathrm{mM}$ citrate buffer ( $\mathrm{pH}$ 6.0) for 40 minutes. After rinsing in PBS and incubation with $2 \%$ BSA in PBS at room temperature for 60 minutes, incubation with a mixture of rabbit anti-HATKTAK IgG (x1,000 diluted) and murine anti-CD 61 monoclonal IgG (Dako Japan, Tokyo, Japan) (x100 diluted) at $4{ }^{\circ} \mathrm{C}$ overnight was performed followed by incubation with a mixture of Alexa Fluor 594-conjugated anti-rabbit IgG (x200 diluted) and Alexa Fluor 488-conjugated anti-murine antibody (x200 diluted) (Molecular Probes, Eugene, OR, USA). The fluorescent signals were viewed under a confocal microscope (Bio-Rad Radiance 2100). As a control experiment, an identical immunohistochemical procedure with omission of the primary antibodies was performed.

\subsection{Assay of concentrations of Apo CIII, apolipoprotein AI (Apo AI) and apolipoprotein B100}

Concentrations of Apo CIII, Apo AI and Apo B100 in the platelet lysate were assayed by ELISA using commercially available assay kits for Apo CIII (AssayPro, St. Charles, MO), Apo AI (Mabtech AB, Nacka Strand, Sweden) and Apo B100 (Mabtech AB), respectively. 

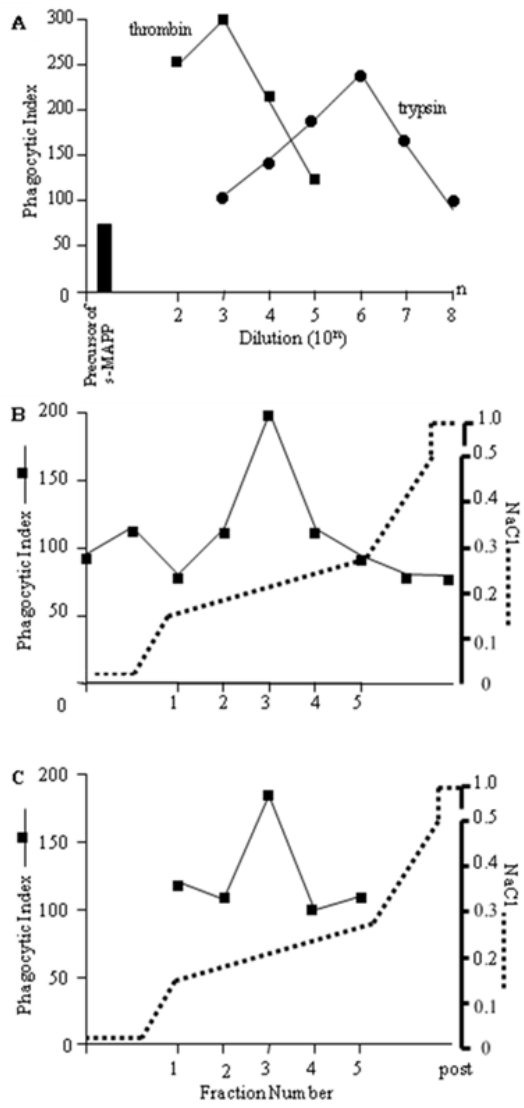

G

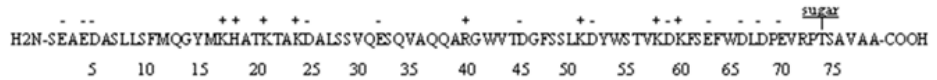

Figure 1. Characterization of the LMW activator produced from Apo CIII. A. MAPP formation using Apo CIII. To prepare LMW activator, $10 \mu \mathrm{g} / \mathrm{ml}$ Apo CIII was treated with $0.1 \mathrm{unit} / \mathrm{ml}$ thrombin or 1 unit $/ \mathrm{ml}$ trypsin, and then diluted serially $x 10$. The activity of each diluted sample as LMW activator was examined. B. MONO S cation exchange chromatography of thrombin-treated Apo CIII (10 $\mu \mathrm{g})$. MAPPs were generated using each fraction at a dilution of $\times 10^{2}$ and one of the precursors of MAPPs. C. MONO $S$ cation exchange chromatography of trypsin-treated apolipoprotein CIII $(10 \mu \mathrm{g} / \mathrm{ml})$. MAPPs were generated using each fraction at a dilution of $x 10^{4}$ and one of the precursor of MAPPs. D. Superdex peptide gel filtration of thrombin-treated Apo CIII $(10 \mu \mathrm{g} / \mathrm{ml})$. Formation of MAPPs was achieved using each fraction at a dilution of $\times 10^{2}$ and one of the precursors of MAPPs. E. Superdex peptide gel filtration of trypsin-treated Apo C-III $(10 \mu \mathrm{g} / \mathrm{ml})$. Formation of MAPPs was achieved using each fraction at a dilution of $\times 10^{4}$ and one of the precursors of MAPPs. F. Molecular weight determination of the LMW activator with comparisons with those of Apo CIII-derived peptides. G. Amino acid sequence of Apo CIII, distributions of basic (+) and acidic (-) amino acids and sugar binding amino acid (T74) in Apo CIII. The amino acid sequence is cited from the database of GenPex (NCBI Protein DataBase, http://www.ncbi.nlm.nih.gov/protein). A, B, C, D and E were performed using the precursor of s-MAPP. 


\subsection{Statistics}

In this study, the differences were analyzed using Mann-Whitney test, Kruskal-Wallis test, paired $t$ test or Wilcoxon test. A $P$ value of less than 0.05 was considered significant.

\section{Results}

\subsection{LMW activator is considered to be HATKTAK}

As it was shown in a previous study that both thrombin and trypsin could produce a substance with LMW activator activity from platelet lysate [27] and that incubation of Apo CIII with thrombin resulted in production of LMW activator, Apo CIII was treated with thrombin or trypsin and the LMW activator activities produced were compared to determine whether LMW activators produced by these enzymes are the same substance. Both thrombin and trypsin could produce LMW activator activity, but 1 unit/ml trypsin could produce LMW activator 100 to 1,000 times more effectively than $0.1 \mathrm{unit} / \mathrm{ml}$ thrombin (Figure 1A).

The LMW activator activities prepared using thrombin and trypsin appeared at the same $\mathrm{NaCl}$ concentration of the MONO S cation exchange chromatography $(0.2 \mathrm{M} \mathrm{NaCl}, \mathrm{pH} 8.0)$ (Figure 1B and Figure 1C, respectively), and at the same elution volume $(16 \mathrm{ml})$ of the Superdex peptide gel filtration (Figure 1D and Figure 1E, respectively), which corresponds to the approximate molecular size of $800 \mathrm{Da}$ (Figure 1F). These findings suggest that the two LMW activators are the same substance.

The fact that LMW activator can be produced by trypsin digestion of Apo CIII suggests that the $\mathrm{C}$ terminal of LMW activator consists of K or R [35]. LMW activator is suggested to be one of the basic peptides that would appear after trypsin digestion of Apo CIII (S1-K24, H18-R40, H18-K24, H18-K21, T22-K24) (see Figure 1G for the sequence of Apo CIII, distribution of basic and anionic amino acids and the sugar binding amino acid). These peptides were artificially produced and filtered on the Superdex peptide column to use as molecular size markers. The optical peaks of OPA fluorescence and LMW activator activity by H18-K24 (HATKTAK) appeared at the same elution volume of Superdex peptide gel filtration corresponding to Apo CIII-derived LMW activator (Figure 1D, 1E, 2A and 2B). In MONO S cation exchange chromatography, the action of HATKTAK as LMW activator was recovered at the fraction with the same $\mathrm{NaCl}$ concentration $(0.2 \mathrm{M} \mathrm{NaCl}, \mathrm{pH} 8.0)$ (Figure $2 \mathrm{C}$ ) as the Apo CIII-derived LMW activator (Figure 1B and 1C).

\subsection{Only HATKTAK among examined peptides showed MAPP-forming activity}

Among the peptides used for calibration on Superdex peptide gel filtration (Figure 1F), H18-R40 and H18-K21 were separated in the fractions near to HATKTAK (LMW activator). The activity of these peptides as LMW activators were examined. Only HATKTAK showed LMW activator activity with a peak at $1 \mathrm{nM}$ (Figure 2D). Then, some Apo CIII-derived peptides that contain part or all of H18-K24 were examined for their LMW activator activity. All of them were used at a concentration of $1 \mathrm{nM}$. Only HATKTAK showed MAPP-forming activity (Table 1). 

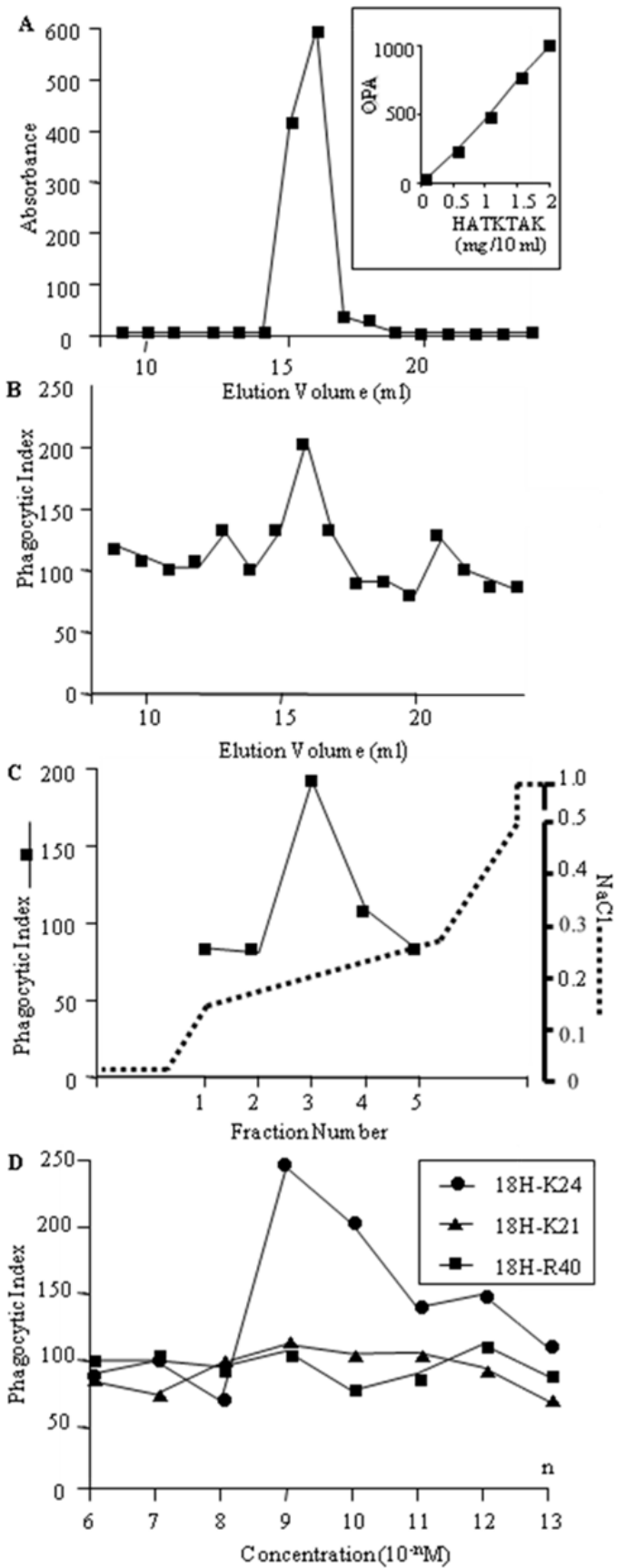

Figure 2. HATKTAK functions as the LMW activator. A. Superdex peptide gel filtration of HATKTAK $(200 \mu \mathrm{g}, 0.2 \mathrm{ml})$. The peptide concentration of each fraction was measured by the OPA fluorescent 
method. The inset shows the standard graph for the assay of HATKTAK. B. MAPP formation using fractions of Superdex peptide gel filtration of HATKTAK $(200 \mu \mathrm{g}, 0.2 \mathrm{ml})$ at a dilution of $x 10^{6}$ by incubation with one of the precursors of MAPPs. C. MONO S cation exchange chromatography of 1 $\mu \mathrm{g} / \mathrm{ml}$ HATKTAK. MAPPs were generated by incubation of each fraction from the chromatography at a dilution of $\times 10^{4}$ and one of the precursors of MAPPs. D. MAPP formation using peptides containing part of the sequence of apolipoprotein CIII. Each peptide was serially diluted x10 and incubated with one of the precursors of MAPPs. B, C and D were performed using the precursor of s-MAPP.

\begin{tabular}{lcc}
\hline \multicolumn{1}{l}{ Peptides } & Precursor of l-MAPP & Precursor of s-MAPP \\
\hline HATK & - & - \\
HATKT & - & - \\
HATKTA & - & - \\
HATKTAK & + & - \\
HATKTAKD & - & - \\
K & - & - \\
TAK & - & - \\
ATKTAK & - & - \\
KHATKTAK & - & - \\
\hline
\end{tabular}

Table 1. MAPP formation from precursors of MAPPs with peptides derived from K16 to D25 of Apo CIII (KHATKTAKD)

\subsection{LMW activator activity in platelet release products and platelet lysate}

In the next part of the study, the LMW activator activities in variously prepared samples including platelet release products and platelet lysate were compared to determine whether platelets release LMW activator. Platelet release products were prepared using platelets from fresh and stored platelet-rich plasma. The reason why we examined platelet release products prepared from stored platelet-rich plasma along with those prepared from the fresh equivalent is that platelet releasate from stored platelet-rich plasma was expected to contain a higher concentration of LMW activator because platelets lose the precursors of MAPPs during storage [26]. From all of these samples, the low-molecular-weight fraction was separated using PD10 columns, and the activity corresponding to LMW activator was compared by the largest dilution from the original sample for which a phagocytic index higher than 150 was recorded (effective dilution).

$1 \mathrm{ng} / \mathrm{ml}$ HATKTAK and platelet release products from fresh platelets stimulated with 4 $\mathrm{mEq} / \mathrm{l} \mathrm{Ca}{ }^{++}$and 0.1 unit $/ \mathrm{ml}$ thrombin showed an effective dilution of $10^{2}$ to $10^{3}$, whereas fresh platelets stimulated only with $4 \mathrm{mEq} / 1 \mathrm{Ca}^{++}$or $0.1 \mathrm{unit} / \mathrm{ml}$ thrombin for 1 minute showed far lower effective dilution, and platelet release products prepared from platelets after storage for 72 hours and 120 hours in the form of platelet-rich plasma showed extremely high effective dilution $\left(10^{3}\right.$ to $10^{5}$, platelets from platelet-rich plasma stored for 72 hours; $10^{7}$ to $10^{8}$, those stored for 120 hours). None of the platelet lysate produced from platelets in fresh and stored platelet-rich plasma showed LMW activator activity (Figure 3). 
To confirm whether these LMW activator activities were by the same substance, Superdex peptide gel filtrations of the low-molecular-weight fractions of platelet release products prepared from fresh platelets (A), and those stored for 72 hours (B) and 120 hours (C), with stimulation with $0.1 \mathrm{unit} / \mathrm{ml}$ thrombin in the presence of $4 \mathrm{mEq} / 1 \mathrm{Ca}^{++}$were performed. Fractions of the gel filtrations were diluted $\times 10^{2}(\mathrm{~A}), \times 10^{4}(\mathrm{~B})$ and $\times 10^{6}(\mathrm{C})$. All of these samples showed LMW activator activity at the fraction corresponding to HATKTAK (compare Figure 4A, 4B and 4C with Figure 2B).

\subsection{Inhibition of LMW activator activity by anti-HATKTAK antibody}

Then, to obtain evidence of the existence of HATKTAK in the platelet release products, actions of an anti-HATKTAK rabbit antibody against LMW activator activity were examined.

Platelet release products

Fresh platelets

$\mathrm{Ca}^{++}, 1 \mathrm{~min}$ in plastic

Fresh platelets

thrombin

Fresh platelets

$\mathrm{Ca}^{++}+$thrombin

Stored platelets $(72 \mathrm{~h})$

$\mathrm{Ca}^{++}+$thrombin

Stored platelets $(120 \mathrm{~h})$

$\mathrm{Ca}^{++}+$thrombin

Platelet lysate

Fresh platelets

Stored platelets (72 h)

Stored platelets $(120 \mathrm{~h})$

HATKTAK (1 ng/ml)

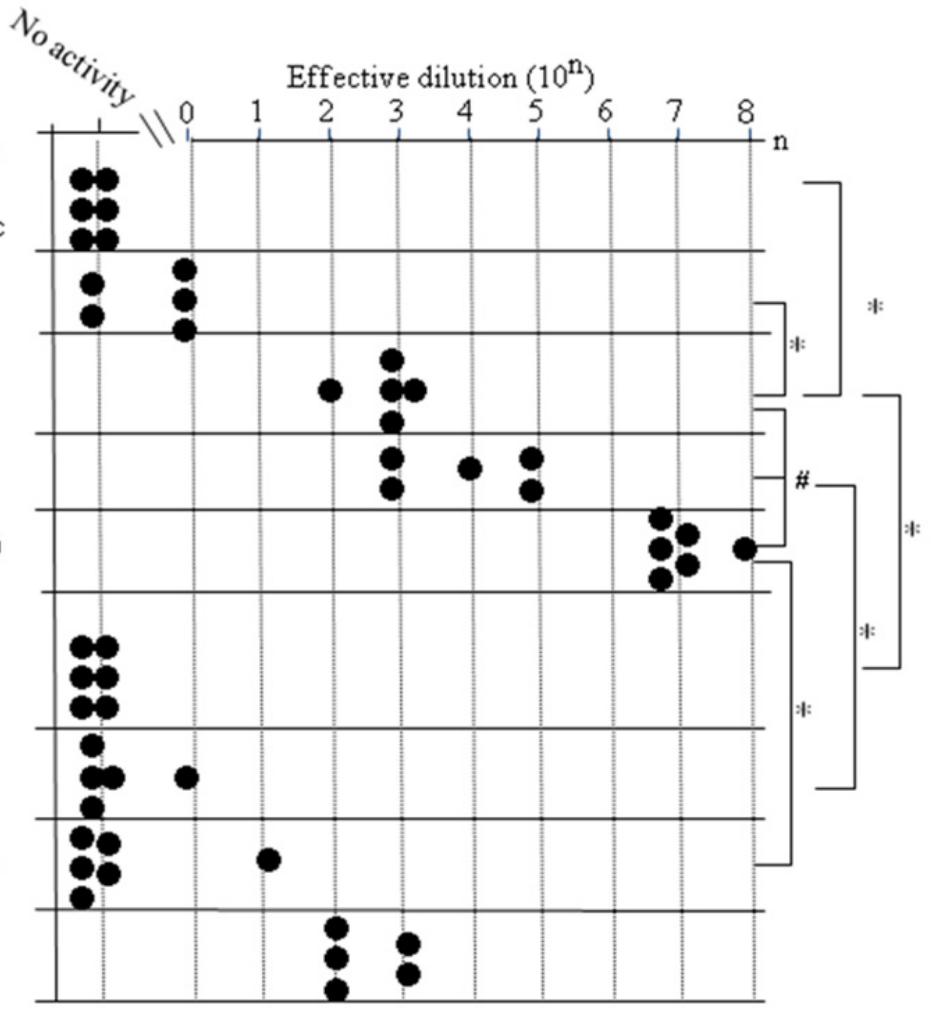

Figure 3. Comparisons of effective dilution of LMW activator in platelet release products and platelet lysate prepared using platelets that were obtained from platelet-rich plasma stored for 0 hours, 72 hours and 120 hours. A black circle means a case with the effective dilution of LMW activator indicated by the horizontal axis. ${ }^{*}, P<0.01$ with the Mann-Whitney test, and \#, $P<0.01$ with the Kruskal-Wallis test. All experiments were performed using the precursor of s-MAPP. 

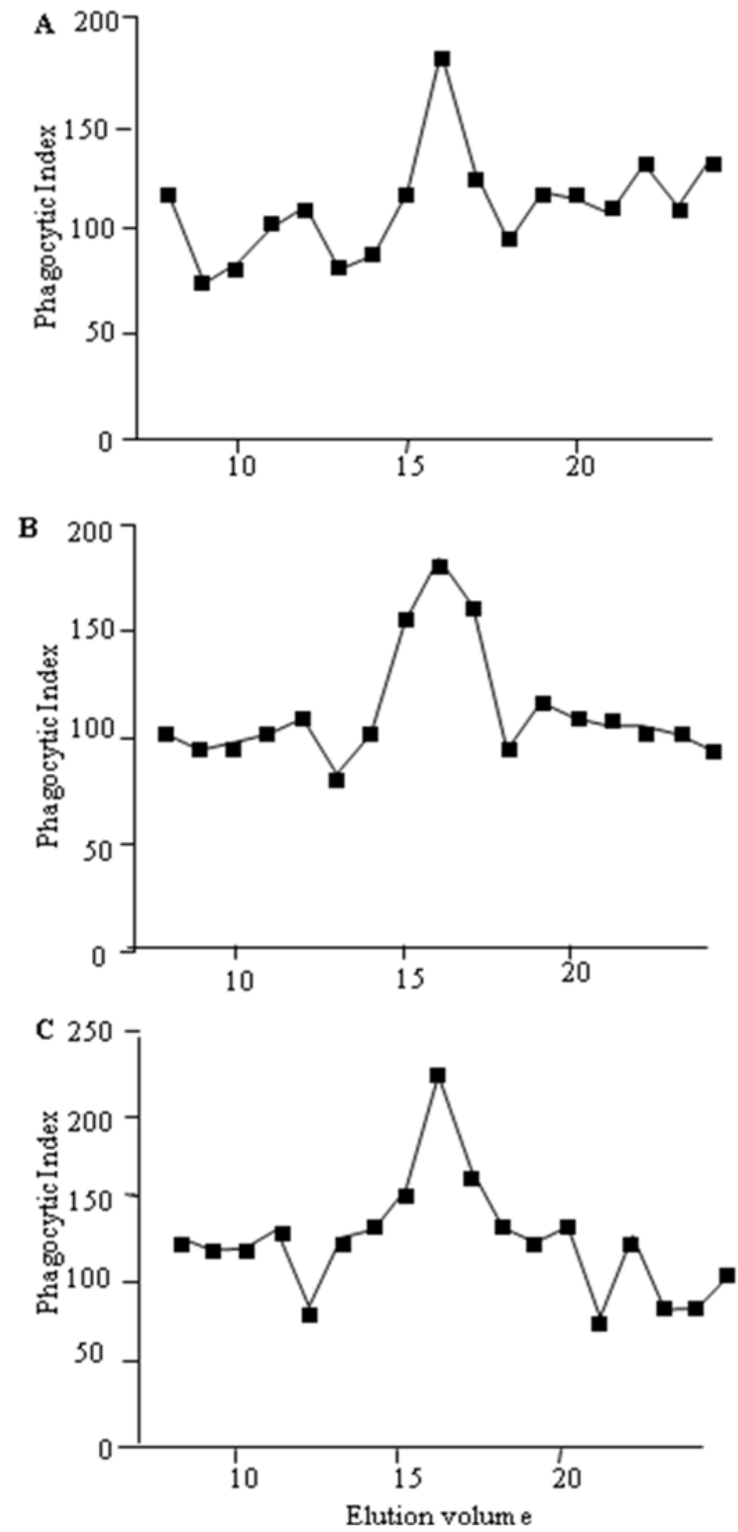

Figure 4. Comparisons of molecular size of LMW activator in platelet release products prepared using (A) platelet release products using fresh platelets stimulated with 0.1 unit $/ \mathrm{ml}$ thrombin in the presence of $\mathrm{Ca}^{++}$; (B) platelet release products using platelets stored for 72 hours stimulated with $0.1 \mathrm{unit} / \mathrm{ml}$ thrombin in the presence of $\mathrm{Ca}^{++} ;(\mathbf{C})$ platelet release products using platelets stored for 120 hours stimulated with $0.1 \mathrm{unit} / \mathrm{ml}$ thrombin in the presence of $\mathrm{Ca}^{++}$. The LMW activator activity of each fraction was examined at dilutions of $\times 10^{2}(\mathbf{A}), \times 10^{3}(\mathbf{B})$ and $\times 10^{6}(\mathbf{C})$. All experiments were performed using the precursor of s-MAPP. 
ELISA revealed that anti-HATKTAK reacted strongly to H18-K24 (HATKTAK), K17-K24 and S1-K24 of Apo CIII, but very weakly to Apo CIII and H18-D25 of Apo CIII, whereas anti-Apo CIII goat antibody showed a positive reaction only to Apo CIII (Figure 5A and 5B, respectively).
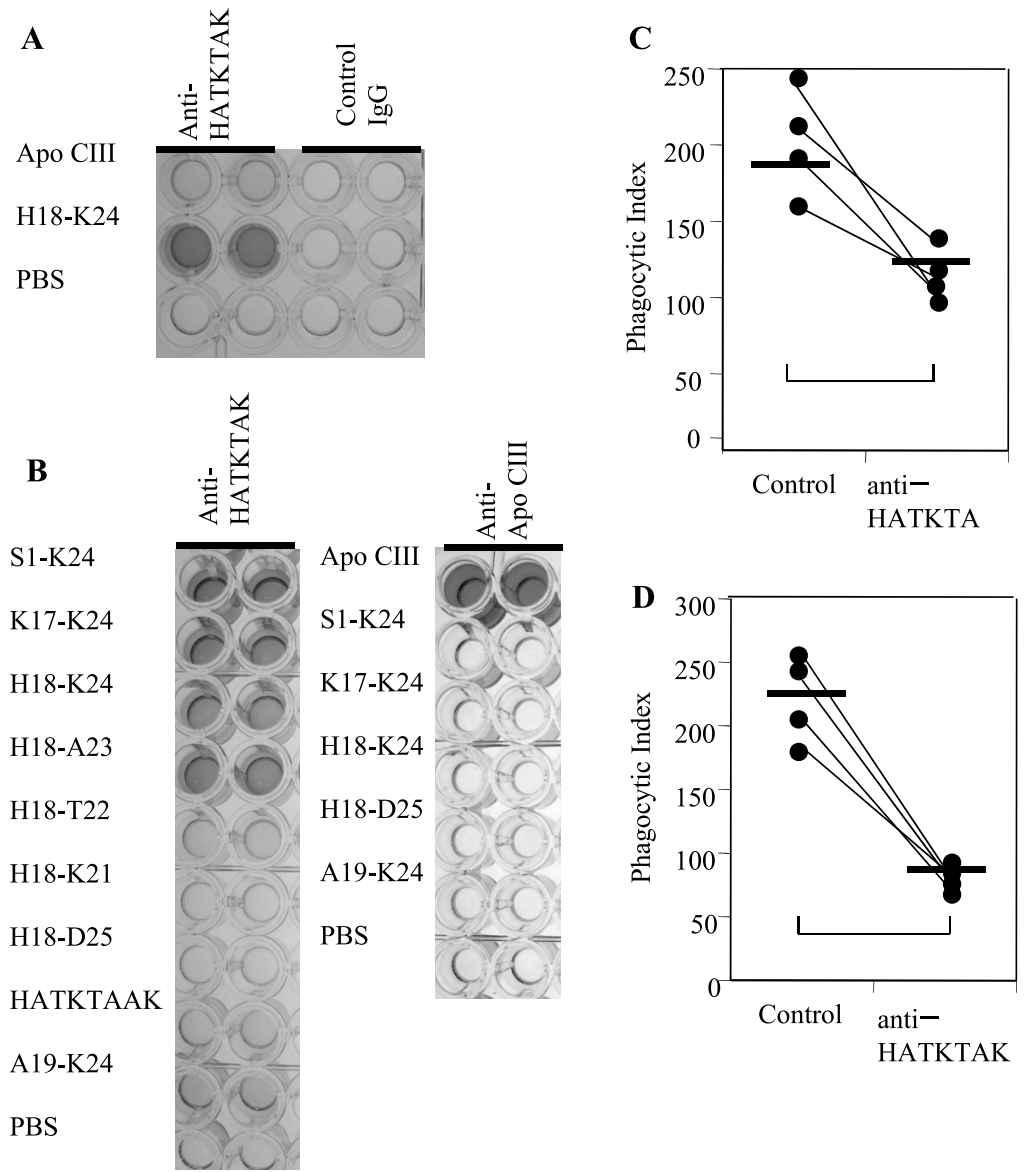

Figure 5. Effects of anti-HATKTAK antibody on LMW activator activity. A, ELISA using antiHATKTAK rabbit IgG and control rabbit IgG against Apo CIII and H18-K24 of Apo CIII. B, ELISA using anti-HATKTAK rabbit IgG and anti-Apo CIII gout IgG against peptide associated with Apo CIII, HATKTAAK and Apo CIII. C, Effects of control rabbit IgG and anti-HATKTAK rabbit IgG on LMW activator activity in the low-molecular-weight fraction of platelet release products prepared from platelets of fresh platelet-rich plasma. D, Effects of control rabbit IgG and anti-HATKTAK rabbit IgG on LMW activator activity in the low-molecular-weight fraction of platelet release products prepared using platelets of platelet-rich plasma stored for 120 hours. In $\mathbf{C}$ and $\mathbf{D}$, figures reveal the result of experiments using the precursor of s-MAPP. The bars in the figure show the average. *,$P<0.01$; ${ }^{* *}, P<0.05$ by paired $t$ test.

Both LMW activators derived from fresh platelet and platelets stored for 120 hours were inhibited by the anti-HATKTAK IgG antibody (Figure 5C and 5D, respectively). 


\subsection{Evidence of existence of HATKTAK by mass spectrometry}

In mass spectrometry, HATKTAK showed m/z 756 (Figure 6A). Although it was impossible to show the existence of a substance with m/z 756 in thrombin-digested Apo CIII $(10 \mu \mathrm{g} / \mathrm{ml})$ and in platelet release products released from fresh platelets and those stored for 72 hours (data not shown), a substance with $\mathrm{m} / \mathrm{z} 756$ was detected in the trypsin-treated Apo CIII (10 $\mu \mathrm{g} / \mathrm{ml}$ ) (Figure $6 \mathrm{~B}$ ) and platelet release products prepared from platelets stored for 120 hours (Figure 6C).
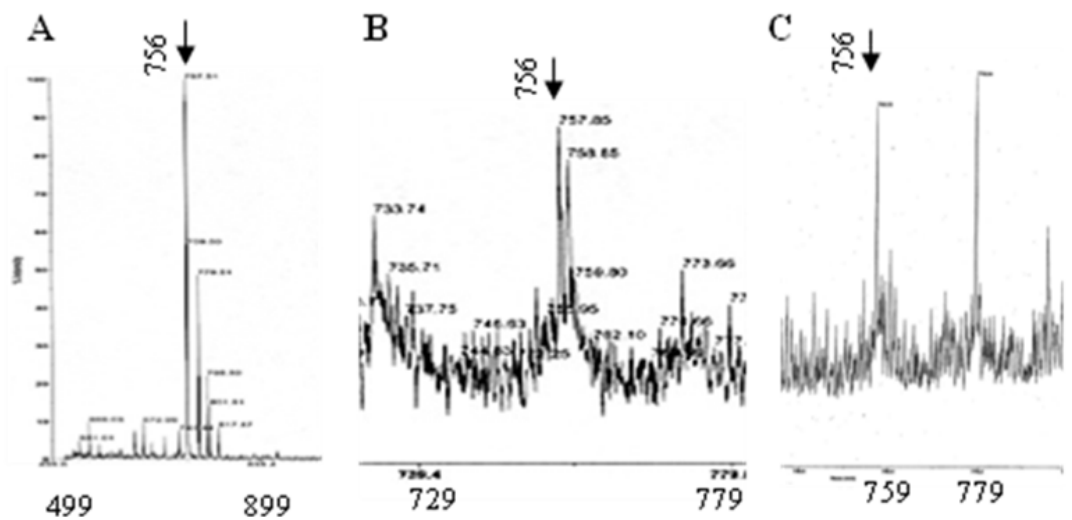

Figure 6. Mass spectrometry of HATKTAK, trypsin-digested Apo CIII and platelet release products from platelets of platelet-rich plasma stored for 120 hours. A, $1 \mathrm{mg} / \mathrm{ml}$ HATKTAK in PBS; B, $10 \mu \mathrm{g} / \mathrm{ml}$ Apo CIII digested with 1 unit/ml trypsin; and $\mathbf{C}$, platelet release products using platelets of platelet-rich plasma stored for 120 hours. Platelets were stimulated with $0.1 \mathrm{unit} / \mathrm{ml}$ thrombin in the presence of $\mathrm{Ca}^{++}$.
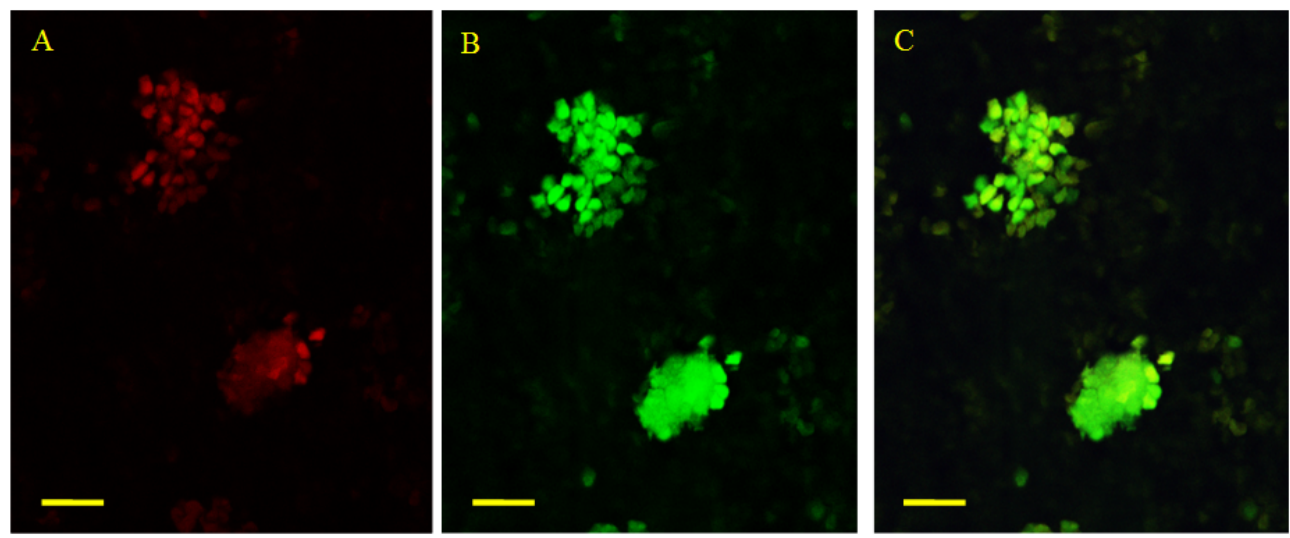

Figure 7. Confocal microscopic images of double staining for the anti-HATKTAK antibody (visualized as red in A), and the anti-CD61 antibody (visualized as green in B). The colocalization of two antibodies is indicated by the conversion of green and red to yellow (C). Scale bar indicate $20 \mu \mathrm{m}$. 


\subsection{Immunohistochemical evidence of existence of HATKTAK in activated platelets}

To show the existence of HATKTAK in activated platelets, an immunohistochemical study of the blood coagula was performed. Platelets in the coagula showed positive reactions with both anti-HATKTAK and anti-CD 61 antibodies simultaneously (Figure 7).

\subsection{Increases in concentrations of Apo CIII, Apo AI and Apo B100 in the platelet lysate}

As the LMW activator activity in the products released from platelets increased markedly if stored platelets were used, concentrations of Apo CIII, Apo AI and Apo B100 in the platelet lysate prepared from platelet-rich plasma stored for 120 hours at $20^{\circ} \mathrm{C}$ were compared with those prepared from fresh equivalent plasma to determine whether lipoproteins are internalized by platelets during storage of platelet-rich plasma. All of Apo CIII, Apo AI and Apo B100 in the lysate from 120 hour-stored platelets were high $(20 \mathrm{ng} / \mathrm{ml}, 18 \mathrm{ng} / \mathrm{ml}$ and 252 $\mathrm{ng} / \mathrm{ml}$ in average, respectively), whereas those from platelet-rich plasma before storage were very low $(1.4 \mathrm{ng} / \mathrm{ml}, 0.6 \mathrm{ng} / \mathrm{ml}$ and $130 \mathrm{ng} / \mathrm{ml}$ on average, respectively) (Figure 8).
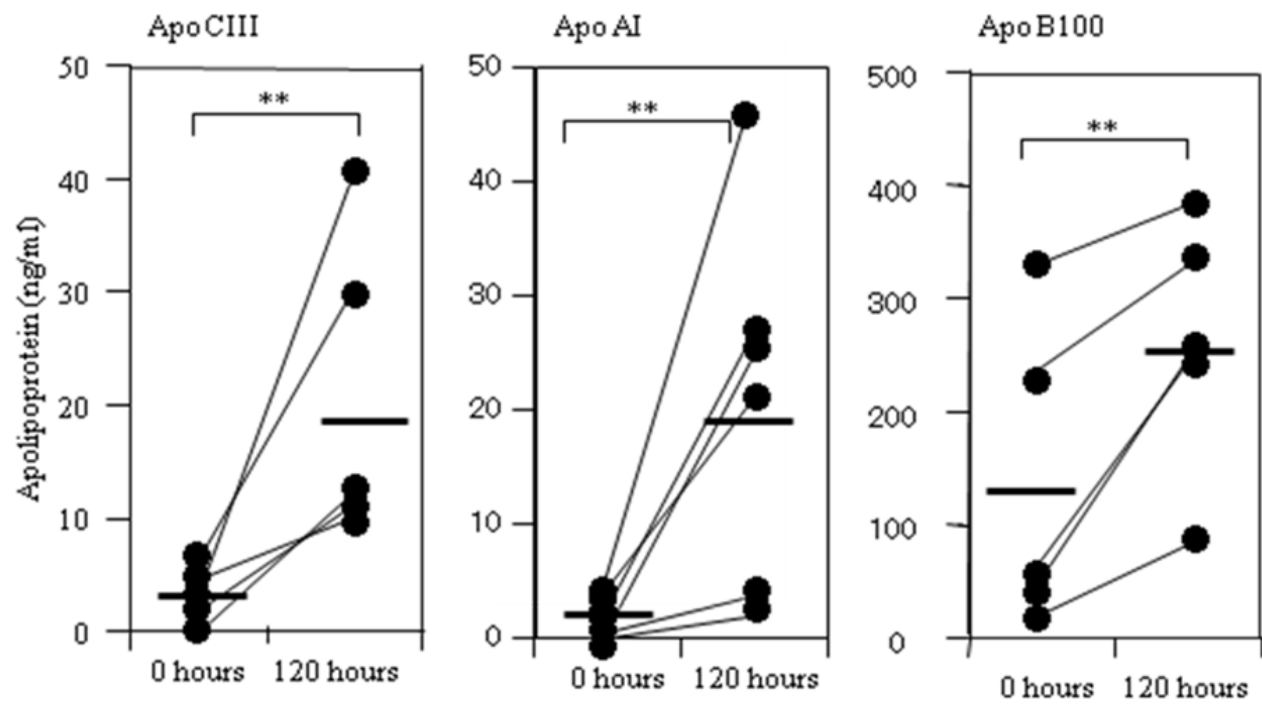

Figure 8. Concentrations of Apo CIII, Apo AI and Apo B100 in the platelet lysate. Concentrations of Apo CIII, Apo AI and Apo B100 in the platelet lysate, which were prepared from platelet-rich plasma before ( 0 hours) and after storage for 120 hours (120 hours), were determined using commercially available ELISA kits. The bars in the figure show the average. ${ }^{* *}, P<0.05$ by Wilcoxon test.

\section{Discussion}

Since we found MAPPs in platelet release products, we have investigated their structure and the mechanisms of production and release by platelets. When fresh platelets are 
stimulated by thrombin, collagen or centrifugation in the presence of $\mathrm{Ca}^{++}$, MAPPs are released. When platelets are stored longer than 72 hours in the form of platelet-rich plasma, they lose the capacity to release MAPPs. It is speculated that this is because platelets lose their contents of thrombin and precursors of MAPPs during storage in the form of plateletrich plasma. They recover the MAPP-releasing function by washing with PBS and incubation with plasma-derived precursors of MAPPs and thrombin in the presence of $\mathrm{Ca}^{++}$[26]. This suggests that some platelet factor other than thrombin and the precursors of MAPPs is necessary for MAPP production. To analyze this factor, the action of the platelet lysate obtained by freeze-thaw of stored platelets on MAPP formation was investigated. By incubation of the platelet lysate with thrombin and the plasma-derived precursors, it was possible to produce MAPPs. When producing MAPPs using plasma-derived precursors and the platelet lysate, we found that trypsin instead of thrombin can produce MAPPs [27].

After we found that the plasma precursors of MAPPs are dimer and tetramer transferrins [28], we produced precursors of MAPPs by glutaraldehyde treatment of commercially available holo-transferrin and used them to produce MAPPs in vitro.

In the former study [30], we found that Apo CIII associated with the high-density lipoprotein in platelet lysate can be the source of LMW activator. In this study, we compared the LMW activators produced from Apo CIII by the actions of thrombin and trypsin. It was revealed that both LMW activators appeared in the fractions of the same elution volume of Superdex peptide gel filtration and in the fractions with the same $\mathrm{NaCl}$ concentration of MONO S cation exchange chromatography. These findings suggest that the LMW activators produced by thrombin and trypsin are the same substance. The fact that trypsin can form LMW activator suggests that LMW activator is a peptide with a C-terminal amino acid of lysine or arginine and that the N-terminal is an amino acid next to lysine or arginine in the amino acid sequence of Apo CIII. It can be asserted that the action of thrombin in LMW activator formation occurs by its trypsin-like activity, although the activity of thrombin to release LMW activator from Apo CIII is far lower than that of trypsin. Cation exchange chromatography in this study revealed that the LMW activator is cationic, suggesting that it is a peptide rich in basic amino acids. In Apo CIII, K17 to $\mathrm{K} 24$ is a region rich in basic amino acids, containing four basic amino acids among eight amino acids in total. Several candidate peptides for LMW activator that contain all or part of the K17 to K24 peptide were raised. By comparing the elution volumes of peaks of these peptides with that of LMW activator function on Superdex peptide gel filtrations and by examining MAPP formation using these peptides, it was strongly suggested that HATKTAK is the LMW activator.

As for the reaction of thrombin, the most abundant natural substrate of thrombin is fibrinogen [36], whereas O'Mullan et al. [37] reported that the action of thrombin to various proteins including Apo CIII is more variable and various peptides cleaved from Apo CIII appear by the action of thrombin on Apo CIII. Our study revealed that the trypsin-like thrombin activity that digests Apo CIII to release HATKTAK is weak, but does in fact exist. We have shown that GP-1b $\alpha$-bound thrombin functions in MAPP production [29]. GP-Ib $\alpha$ is 
a high-affinity thrombin receptor on platelets [38-39]. Binding of thrombin with GP-1b might enhance the trypsin-like activity of thrombin in platelets.

To confirm that LMW activator, HATKTAK, is produced by the platelets, we examined the LMW activator activity in the low-molecular-weight fractions of variously prepared platelet release products. We found that, in the platelet release products prepared using $4 \times 10^{5} / \mu \mathrm{l}$ fresh platelets $\left(0.1 \mathrm{unit} / \mathrm{ml}\right.$ thrombin in the presence of $\left.4 \mathrm{mEq} / \mathrm{l} \mathrm{Ca}^{++}\right)$, the LMW activator involved is as much as $1 \mathrm{ng} / \mathrm{ml}$ HATKTAK.

By storage of the platelet-rich plasma, release of LMW activator from platelets induced by thrombin in the presence of $\mathrm{Ca}^{++}$increased prominently. It was suggested that the concentration of LMW activator released from platelets stored for 120 hours is 10,000 times as much as that from fresh platelets.

One reason for the tremendous increase in LMW activator, HATKTAK, in the released products during storage is probably the loss of the precursor of MAPPs during storage, as shown in a previous report [26]. It is speculated that, in activated fresh platelets, LMW activator (HATKTAK) is produced as much as in stored platelets, but it decreases markedly because precursors of MAPPs remove it in fresh platelets. Another possible reason is that lipoproteins, apolipoproteins or fragments of apolipoproteins might be transported at high levels into platelets from the plasma during storage. In fact, it was shown that the concentrations of Apo CIII and Apo A1 in the platelet lysate increased markedly after storage of platelets in the form of platelet-rich plasma, but this was still too small to explain the observed increase in the effective dilution by as much as 1,000 times.

Indirect ELISA of the platelet release products using anti-HATKTAK antibody was undertaken to prove the existence of HATKTAK. The results were satisfactory if synthesized pure peptides were used, and it was shown that the anti-HATKTAK rabbit antibody reacted positively to Apo CIII-derived peptides with C-terminal HATKTAK. However, we have not succeeded in establishment of a method to analyze HATKTAK in platelet release products. It is postulated that some substances derived from the platelet release products interfere with the adherence of HATKTAK on the wall of microtiter plate. Therefore, we examined the effect of the antibody on the LMW activator function. It was confirmed that the antiHATKTAK antibody cancels the activity of the LMW activator in the platelet release products from fresh platelet-rich plasma and that stored for 120 hours. Mass spectrometry study revealed the presence of a substance corresponding to HATKTAK (m/z 756) in the platelet release products from platelets stored for 120 hours. Immunohistochemistry of the blood coagula revealed the existence of platelets with double-positive reaction to antiHATKTAK and anti-CD61 antibodies. These findings strongly suggest that the LMW activator is HATKTAK and is produced and released by platelets.

A schematic illustration of the probable mechanism of production and release of MAPPs and HATKTAK by platelets is depicted in Figure 9.

At present, the mechanism of how MAPP contributes to neutrophilic phagocytosis enhancement after binding to neutrophils is not known. Because MAPPs possess transferrin molecules and anti-transferrin receptor antibody inhibits the action of MAPPs [28], it is 
suggested that the action of MAPPs occurs via the transferrin receptor. As shown previously, treatment of neutrophils with MAPP does not result in an increase in the number of Fc receptors on neutrophils [40]. This suggests that the effect of MAPP is to strengthen the affinity of $\mathrm{Fc}_{\mathrm{c}}$ receptors with the $\mathrm{Fc}_{\mathrm{c}}$ portion of IgG and to internalize more foreign materials inside the cell. The necessity of HATKTAK for MAPP function suggests that the transferrin receptor might have a site for binding to both transferrin and HATKTAK, and the fact that MAPPs consist of multimers (dimer and tetramer) of transferrin suggests that transferrin receptors must be fixed at an appropriate distance when MAPPs induce enhancement of phagocytosis via the Fc receptors. The mechanisms of how the transferrin receptor, which is stimulated by HATKTAK and transferrin, transfers the information to $\mathrm{Fc}$ receptors remain to be elucidated.

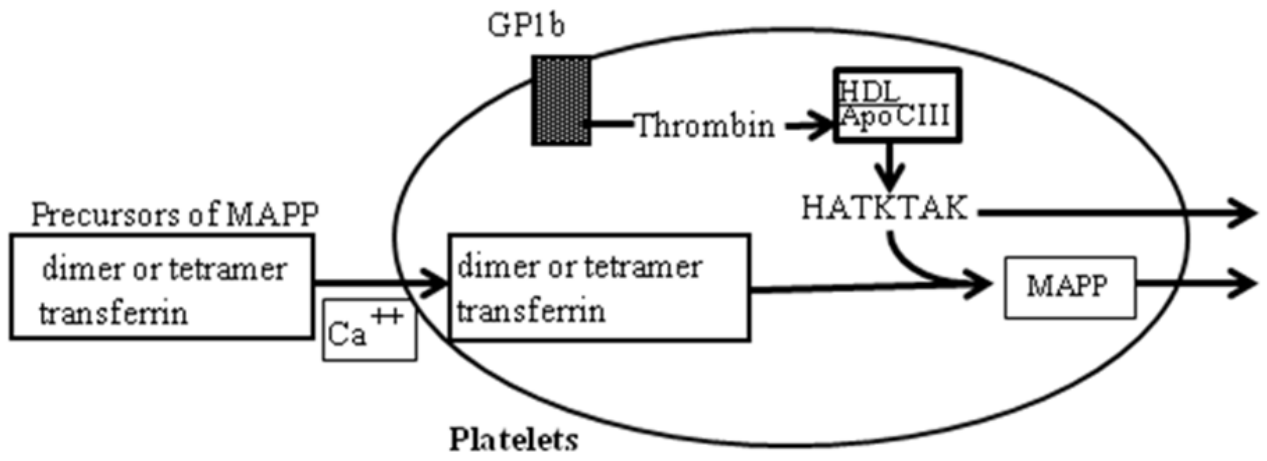

Figure 9. A schematic illustration of the mechanism of production of MAPPs and HATKTAK in platelets and their subsequent release.

In conclusion, we could show that HATKTAK is the LMW activator, which is produced in activated platelets and activates MAPPs, and that the residual HATKTAK is released with other platelet-related substances including MAPPs.

\section{Author details}

Haruhiko Sakamoto ${ }^{1,}$, Masaki Ueno ${ }^{1}$, Wu Bin ${ }^{1,2}$, Yumiko Nagai ${ }^{3}$, Kouichi Matsumoto ${ }^{1}$, Takao Yamanaka ${ }^{1,4}$ and Sumiko Tanaka ${ }^{1}$

${ }^{1}$ Inflammation Pathology, Department of Pathology and Host Defense, Faculty of Medicine,

Kagawa University, Kagawa, Japan

2Department of Gynecology and Obstetrics, Second Affiliated Hospital, China Medical University, San Hao Road, Shen Yan, China

${ }^{3}$ Division of Research Instrument and Equipment, Life Science Research Center, Institute of Research Promotion, Kagawa University, Kagawa, Japan

${ }^{4}$ Internal Medicine, Minami-Okayama Medical Center, Incorporated Administrative Agency National Hospital Organization, Okayama, Japan

${ }^{*}$ Corresponding Author 


\section{Acknowledgement}

The authors wish to thank staff and students of the Faculty of Medicine, Kagawa University, for their cooperation in providing their blood for this study.

\section{References}

[1] Sakamoto H, Firkin FC, Chesterman C (1984) Stimulation of leukocyte phagocytic activity by the platelet release reaction. Pathology. 16: 126-130.

[2] Sakamoto H, Firkin F (1984) Characterization of leukocyte phagocytic stimulatory material released by activated human platelets. Br. j. haematol. 57: 47-60.

[3] Ward P, Cunningham TW, McCulloch KK, Phan SH, Powell J, Johnson KJ (1988) Platelet enhancement of ${ }^{-}$response in stimulated human neutrophils. Identification of platelet factor as adenine nucleotide. Lab. invest. 8: 37-47.

[4] Spisani S, Giuliani AL, Cavalletti T, Zaccarini M, Milani L, Gavioli R, Tranello (1992) Modulation of neutrophil function by activated platelet release factor. Inflammation. 16: 147-158.

[5] Ruf A, Schlenk RF, Maras A, Morgenstern E, Patscheke H (1992) Contact-induced neutrophil activation by platelets in human cell suspensions and whole blood. Blood. 80: 1238-1246.

[6] Tsuji T, Nagata K, Koike J, Todoroki N, Irimura T (1994) Induction of superoxide anion production from monocytes and neutrophils by activated platelets through the Pselectin-sialyl Lewis $X$ interaction. J. leuk. biol. 56: 583-587.

[7] Carulli G, Barsotti G, Cupisiti A (1995) Platelet-neutrophil interactions in uremic patients: effects on neutrophil superoxide anion production and chemiluminescence. Nephron. 69: 248-252.

[8] Zalavary S, Grenegard M, Stendahl O, Bengstsson T (1996) Platelets enhance Fc(gamma) receptor-mediated phagocytosis and respiratory burst in neutrophils: the role of purinergic modulation and actin polymerization. J. leuk. biol. 60: 58-68.

[9] Piccardoni P, Evangelis Vt, A. Piccoli, de Gaetano G, Walz A, Celetti C (1996) Thrombinactivated human platelets release two NAP-2 variants that stimulate polymorphonuclear leukocytes. Thromb. res. 76: 780-785.

[10] Neumann F, Max JN, Gawaz M, Brand K, Ott I, Rokitta C, Sticherling C, Meinl C, May A, Schomig A (1997) Induction of cytokine expression in leukocytes by binding of thrombin-stimulated platelets. Circulation. 95: 2387-2394.

[11] Peters MJ, Dixon G, Kotowicz KT, Hatch DJ, Heyderman RS, Klein NJ (1999) Circulating platelet-neutrophil complexes represent a subpopulation of activated neutrophils primed for adhesion, phagocytosis and intracellular killing. Br. j. haematol. 106: 391-399.

[12] Petersen F, Bock L, Flad HD, Brandt E (1999) Platelet factor 4-incduced neutrophilendothelial cell interaction: involvement of mechanisms and functional cosequences different from those elicited by interleukin-8. Blood. 94: 4020-4028. 
[13] Kirton CM, Nash GB (2000) Activated platelets adherent to an intact endothelial cell monolayer bind flowing neutrophils and enable them to transfer to the endothelial surface. J. lab. clin. med. 136: 303-313.

[14] Brandt E, Petersen F Ludwig A, Ehlert JE, Bock L, Flad HD (2000) The betathromboglobulins and platelet factor 4: blood platelet-derived CXC chemokines with divergent roles in early neutrophil regulation. J. leuk. biol. 67: 471-478.

[15] MacGarrity ST, Heyers TM, Webster RO (1988) Inhibition of neutrophil functions by platelets and platelet-derived product: Description of multiple inhibitory properties. J. leuk. biol. 44: 93-100.

[16] Dallegri F, Ballestero, Ottonello AL, Patrone F (1989) Platelets as scavengers of neutrophil derived oxidants: A possible defence mechanism at sites of vascular injury. Thromb.haemost. 61: 415-418.

[17] Moon DG, Der Zee HV, Weston LK, Gudewicz PW, Fenton JW, Kaplan JE (1990) Platelet modulation of neutrophil superoxide anion production. Thrombosis. haemost. 63: 91-96.

[18] Naum C, Kaplan SS, Basford RE (1991) Platelet and ATP prime $\mathrm{O}_{2}^{-}$generation at high concentration. J. leuk. biol. 49: 83-89.

[19] Bengtsson T, Zalavary S, Stendahl O, Genegard M (1996) Release of oxygen metabolites from chemoattractant-stimulated neutrophils is inhibited by resting platelets: role of extracellular adenosine and actin polymerization. Blood. 1996: 87: 4411-4423.

[20] Jancinova V, Drabikova K, Nosal R, Danihelova E (2000) Platelet-dependent modulation of polymorphonuclear leukocyte chemiluminescence. Platelets. 11: 278-285.

[21] Losche W, Temmler U, Redlich H, Vickers J, Krause S, Spangenberg P (2001) Inhibition of leukocyte chemiluminescence by platelets: role of platelet-bound fibrinogen. Platelets. 12: 15-19.

[22] Reinisch CM, Dunzendorfer S, Pechlaner C, Ricevuti G, Wiedermann CJ (2001) The inhibition of oxygen radical release from human neutrophils by resting platelets is reversed by administration of acetylsalicylic acid or clopidogrel. Free. radical. res. 34: 461-466.

[23] Miyabe K, Sakamoto N, Wu YH, Mori N, Sakamoto H (2004) Effect of platelet release products on neutrophilic phagocytosis and complement receptors. Thromb. res. 114: 2936.

[24] Sakamoto H, Ooshima A. (1985) Activation of neutrophil phagocytosis of complement coated and IgG coated sheep erythrocytes by platelet release products. Br. j. haematol. 16: 173-181.

[25] Sakamoto H, Yokoya Y, Ooshima A. (1987) In vitro control of neutrophilic phagocytosis of IgG coated SRBC by macromolecules involved in released products from platelets. J. leuk. biol. 41: 55-62.

[26] Sakamoto H, Ogawa Y, Sakamoto N, Oryu M, Shinnou M, Hirao T (1996) Recovery of macromolecular activators of phagocytosis from platelets (MAPP) producing and releasing function in stored human platelets. Int. j. hematol. 63: 145-148. 
[27] Ogawa Y, Sakamoto H, Oryu, M Shinnou M, SakamotoN, Wu Y, Khatun R, Nishioka M (2000) Production of macromolecular activators of phagocytosis by lysed platelets. Thromb. res. 97: 297-306.

[28] Sakamoto H, Sakamoto N, Oryu M, Kobayashi K, Ogawa Y, Ueno M, Shinnou M (1997) A novel function of transferrin as a constituent of macromolecular activators of phagocytosis from paltelets and their precursors. Biochem. biophys. res. com. 230: 270274.

[29] Sakamoto H, Ueno M, Wu Y, Khatun R, Tanaka S, Miyabe K, Ogawa Y, Onodera M (2000) Glycoprotein Iba-bound thrombin functions as a serine protease to produce macromolecular activators of phagocytosis from platelets. Biochem. biophys. res. com. 270: 377-382.

[30] Sakamoto H, Wu B, Nagai Y, Tanaka S, Onodera M, Ogawa T, Ueno M (2011) Platelet high-density lipoprotein activates transferrin-derived phagoctosis activators, MAPPs, following thrombin digestion. Platelets. 22: 371-379.

[31] Boyle W (1968) An extension of the 51Cr-release assay for the estimation of mouse cytotoxins. Transplant. 62: 761-764.

[32] Avrameas S, Ternyck T (1971) Peroxidase labeled antibody and Fab conjugates with enhanced intracellular penetration. Immunochem. 8: 1175-1179.

[33] Nakamura R, Voller M, Bidwell DE (1986) Chapter 27 Enzyme immune assay: heterogenous and homogeneous system. In: Weir DM, Herzenberg LA, Blackwell C, Herzenberg LA, editors. Handbook of Experimental Immunology. 1 Immunochemistry. Oxford: Blackwell Scientific Publications. pp. 27.1-27.20

[34] Peterson GL (1983) O-phthalaldehyde fluorescent methods for protein quantitation. In: Hirs CHW, Timasheff SN editors. Methods in enzymology 91. New York: Academic Press. pp. 95-98.

[35] Hafon ST, Baird TT and Craik CS. (2004) 452. Trypsin In: Barret AJ, Rawlings ND, Woessner JF, editors. Handbook of Proteolytic Enzymes, Vol 2. Second edition. London: Elsevier Academic Press. pp. 1483-1488.

[36] Brown MA, Sternberg LM, Stenflo J (2004) 510. Thrombin. In: Barret AJ. Rawlings ND, Woessner JF, editors. Handbook of Proteolytic Enzymes, Vol 2. Second edition. London: Elsevier Academic Press. pp. 1667-1672.

[37] O' Mullan P, Draft D, Yi J, Gelfand CA (2009) Thrombin induces broad spectrum proteolysis in human serum samples. Clin. chem. lab. med. 47: 685-693.

[38] Harmon JT, Jamieson GA. (1985) Thrombin binds to a high-affinity approximately 900,000-dalton site on human platelets. Biochem. 24: 58-64.

[39] Jamieson GA (1997) Pathophysiology of platelet thrombin receptors. Thromb. haemost. 78: 242-246.

[40] Sakamoto N, Sakamoto H, Tanaka S, Oryu M and Ogawa Y. (1998) Effects of platelet release products on neutrophilic Fc $\gamma$ receptors. J. leuk. biol. 64: 631-635. 